\title{
Effects of Tourniquet Use on Quadriceps Function and Pain in Total Knee Arthroplasty
}

\author{
David Liu, FRACS ${ }^{1}$, David Graham, MBBS $^{2}$, Kim Gillies, M Hlth.Sc ${ }^{3}$, and R. Mark Gillies, PhD \\ ${ }^{1}$ Gold Coast Centre for Bone and Joint Surgery, John Flynn Private Hospital, Gold Coast; ${ }^{2}$ Department of Orthopedic Surgery, Gold Coast and Robina Hospitals, Gold \\ Coast; ${ }^{3}$ Clinical Data Solutions, Sydney; ${ }^{4}$ University of Sydney, Kolling Institute, Murray Maxwell Biomechanics Laboratory, Royal North Shore Hospital, Sydney, \\ Australia
}

\begin{abstract}
Purpose: A pneumatic tourniquet is commonly used in total knee arthroplasty (TKA) to improve surgical field visualisation but may result in quadriceps muscle ischaemia. We performed this study to analyse the effect of the tourniquet on recovery following TKA.

Materials and Methods: A prospective randomised single-blinded trial was undertaken to examine the effect of the tourniquet on post-operative pain, swelling, blood loss, quadriceps function and outcome following TKA. Twenty patients with osteoarthritis of the knee were randomised to tourniquet or no tourniquet groups. Quadriceps function was assessed using surface electromyography (EMG) during active knee extension.

Results: The no tourniquet group had significantly less pain in the early post-operative period compared to the tourniquet group. There was no difference in Oxford knee score, range of motion, or thigh and knee swelling up to 12 months post-operatively. Quadriceps function, measured by surface EMG, was compromised for the first six months post-surgery by tourniquet use. The radiological cement mantle at the bone prosthesis interface at 12-month follow-up was not affected by the absence of a tourniquet.

Conclusions: We believe that it is safe and beneficial for our patients to routinely perform TKA without a tourniquet.
\end{abstract}

Keywords: Knee, Arthroplasty, Tourniquet, Pain, Quadriceps

\section{Introduction}

Recent emphasis in total knee arthroplasty (TKA) has focused on post-operative pain control and minimising soft tissue trauma during surgery ${ }^{1,2)}$, in an attempt to accelerate recovery, return to function, and reduce hospital stay ${ }^{3}$. Traditionally, a thigh tourniquet is used to prevent intra-operative bleeding and improve surgical field visualisation and ease of surgery ${ }^{4}$. A tourniquet is believed to decrease operative time and blood loss, and provide

Received November 25, 2013; Revised July 13, 2014;

Accepted August 16, 2014

Correspondence to: David Liu, FRACS

Gold Coast Centre for Bone and Joint Surgery, John Flynn Private Hospital, Suite 8A Fred McKay House, 42 Inland Dr, Tugun Queensland 4224, Australia

Tel: +61-7-5598-0205, Fax: +61-7-5598-0205

E-mail: dliu01@bigpond.com

This is an Open Access article distributed under the terms of the Creative Commons Attribution Non-Commercial License (http://creativecommons.org/licenses/by-nc/3.0/) which permits unrestricted non-commercial use, distribution, and reproduction in any medium, provided the original work is properly cited. a dry clean bone surface for optimal cement inter-digitation. Known disadvantages of tourniquet use include ischaemia to the quadriceps muscle ${ }^{5}$, wound complications ${ }^{6}$, neurovascular injury ${ }^{7-9)}$, swelling and bruising ${ }^{10)}$, hidden blood loss ${ }^{5}$, deep venous thrombosis and difficulty with patella tracking assessment ${ }^{11)}$ and the need for lateral release ${ }^{12}$. Less common complications include rhabdomyolysis ${ }^{13,14)}$, and cardio-respiratory effects resulting from free radical release and reperfusion injuries ${ }^{10)}$. In particular, tourniquet use is known to be detrimental to skeletal muscle cell viability ${ }^{15}$. Electromyography (EMG) changes have been demonstrated in the quadriceps muscle following knee surgery with a tourniquet ${ }^{16)}$ and this may affect post-operative recovery and rehabilitation.

Previous studies on tourniquet use in TKA have reported conflicting conclusions. Currently, it remains individual surgeon's preference whether or not to use a tourniquet. Long-term consequence of the tourniquet in TKA on quadriceps strength and quality of the bone prosthetic cement interface is not well known. One potential downside of not using a tourniquet is compromise of cement technique, leading to earlier loosening.

We undertook this study to analyse the effect of the tourniquet 
on recovery following TKA. Our hypothesis is the use of a tourniquet delays recovery because of increased pain, swelling and impaired quadriceps function but improves the quality of the cement interface.

\section{Materials and Methods}

We prospectively randomised twenty patients with osteoarthritis undergoing TKA to either the tourniquet or no tourniquet group. Patients with symptomatic peripheral vascular disease or contra-indication to tourniquet use were excluded. The consolidated standards of reporting trials flow chart is shown in Fig. 1. Institutional Ethics approval was gained prior to study commencement and all patients gave informed consent.

The patients were blinded to group allocation. Randomisation was performed using Excel's random number generator and a random numbers table once the patient was anaesthetised and prepared for commencement of surgery.

All patients underwent TKA by the senior surgeon (Liu) using a standardised technique and prosthesis. All patients received a general anaesthetic without regional blocks or local anaesthesia, in an effort to minimise confounding variables that may influence pain scores. A medial parapatellar approach was used with eversion of the patella. A cemented fixed bearing posterior cruciate-retaining prosthesis was inserted in all patients with resurfac- ing of the patella. A manual cementing technique was used in all patients. Great lengths were taken to clean the bony surfaces with pulsatile lavage and pressurise the cement using a combination of digital pressure and a flat osteotome. An intra-articular drain on low suction was inserted prior to wound closure and removed day one post-operatively. All patients received patient controlled analgesia with morphine sulphate for the first 24 hours. The patients were mobilised day one post-operatively and discharged home when mobilising safely. The same standardised physiotherapy protocol was undertaken in all patients post-operatively. Active and passive range of motion was encouraged without the use of continuous passive motion.

An above knee tourniquet (width $10.5 \mathrm{~cm}$, length $65.5 \mathrm{~cm}$; VBM Medizintechnik GmbH, Sulz, Germany) was placed on all patients during the surgery. If allocated to the tourniquet group, the tourniquet was inflated to $300 \mathrm{mmHg}$ just prior to initial skin incision. This was the standard pressure used at our institution at the time. The tourniquet was deflated following wound closure and application of dressings.

The primary outcome variable was pain during the initial postoperative period measured by the visual analogue pain score. Both the patients and nurses who collected the pain scores were blinded to the patients' group. A score from one to ten was recorded 4 times daily up to discharge and averaged for each day. Pain scores were recorded at 600, 1,200, 1,800, and 2,200 with the

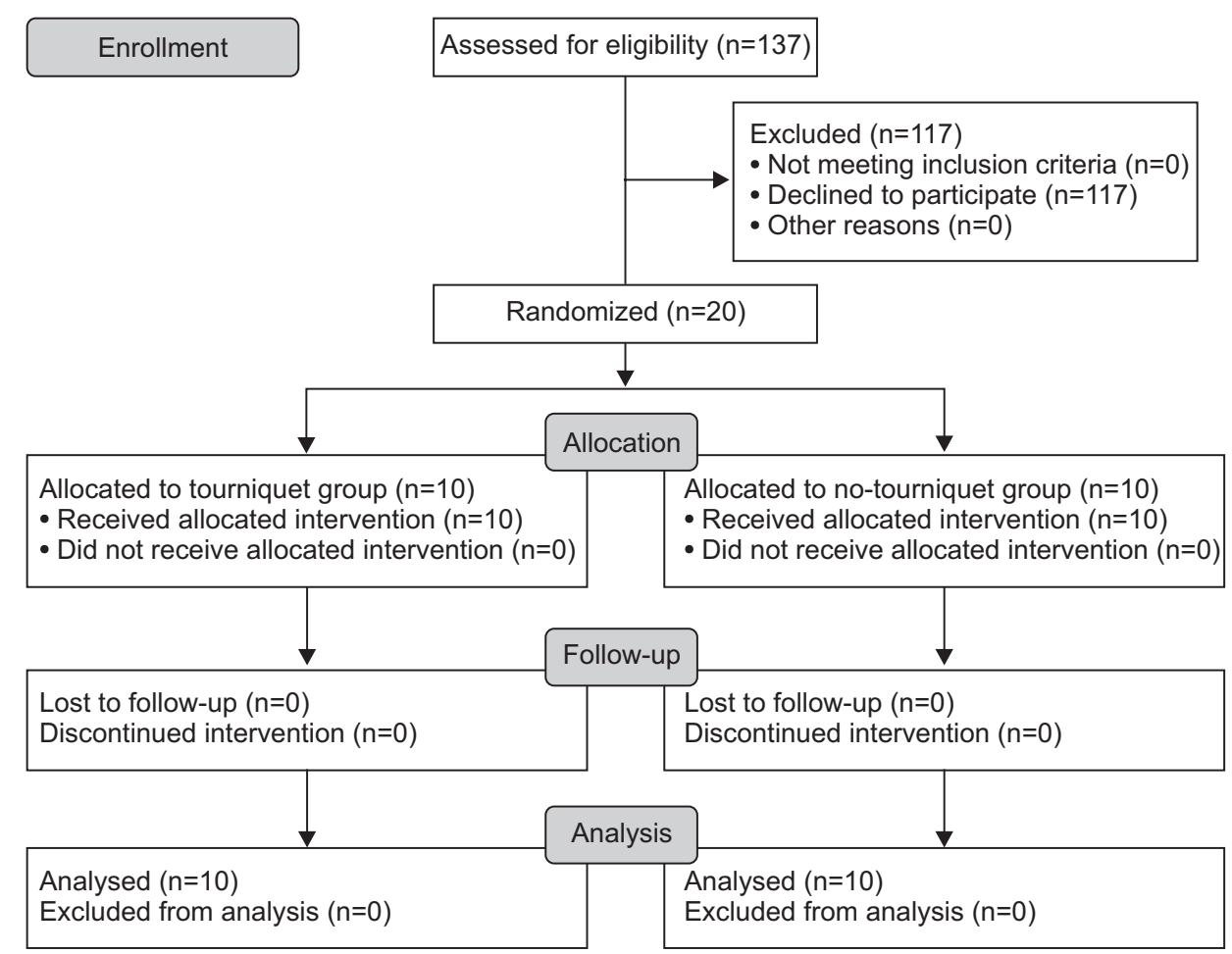

Fig. 1. Consolidated standards of reporting trials (consort) flow diagram for the study. 
patient at rest. These times coincided with the timing of routine panadol administration by the nurses.

Secondary outcome measures included post-operative drainage, transfusion requirements, thigh and knee swelling, morphine requirements, days to discharge, range of motion and Oxford knee score. Thigh and knee swelling was recorded using tape measure at the midpoint of the patella and $10 \mathrm{~cm}$ above the superior pole of the patella. We measured quadriceps function in all the patients during active knee extension against gravity using surface EMG. Surface EMG data was recorded (Pocket EMG; BTS S.p.A., Milano, Italy) for each participant on the day of surgery preoperatively, and at 6 weeks, 6 and 12 months post-operatively. The vastus medialis, rectus femoris and vastus lateralis muscles for both the treated and control knee were recorded. Surface EMG electrodes (3M red dot; 3M Australia, Sydney, Australia) were placed on the skin surface of the muscles. Signal integrity was checked prior to the participant performing ten active knee extensions against gravity with a three second hold with their leg in full extension. The data was downloaded and analysed (Myolab, BTS S.p.A.) by rectifying the surface EMG signals and taking the mean of each of the signals.

Statistical analysis for both parametric and nonparametric data, where the two factors compared were with and without the use of a tourniquet, was performed using a two way analysis of vari-

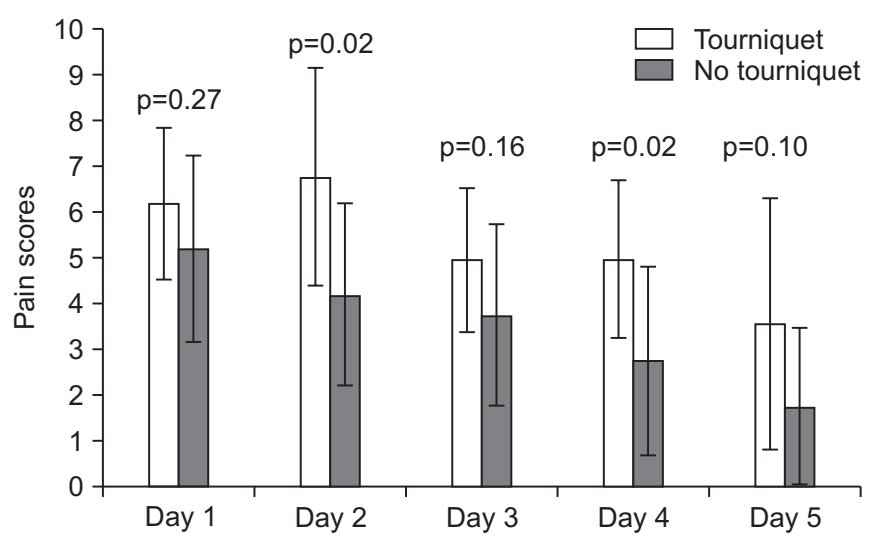

Fig. 2. Average pain scores for the first 5 days post-operatively in the tourniquet and no tourniquet groups. ance (ANOVA; SPSS ver. 13.0 [SPSS Inc., Chicago, IL, USA]). A p-value of 0.05 was taken to be statistically significant.

Anteroposterior and lateral radiographs for each patient were taken at 12 months post-operatively. The digital radiographs were taken under fluoroscopic control to ensure parallelism with the prosthetic-bone interface. The digital radiographs were assessed for cement mantle thickness and radiolucency using the Knee Society radiographic zones by a blinded observer.

\section{Results}

The patient demographics are outlined in Table 1, with no significant difference between the two groups. The average tourniquet time was 83 minutes (range, 67 to 100 minutes). The mean operative time was 13 minutes longer in the no tourniquet group. There were no complications related to not using a tourniquet, such as inadvertent injury to soft tissue structures.

The patients without tourniquet inflation during TKA had less pain as measured by the visual analogue pain score. Fig. 2 displays the average pain scores for the first five days for the two groups. The difference in pain scores between the tourniquet and no tourniquet groups was statistically significant on days 2 and 4 $(\mathrm{p}=0.02)$.

Three patients in the tourniquet group required a blood transfusion whereas none in the no tourniquet group did ( $p=0.05)$. All other clinical outcome measures were not significantly different as shown in Table 2. There was a trend towards a shorter hospital stay in the no tourniquet group but this did not reach statistical

Table 1. Patient Demographics for the Tourniquet and No Tourniquet Groups

\begin{tabular}{lccc}
\hline \multicolumn{1}{c}{ Parameter } & Tourniquet & No tourniquet & p-value \\
\hline Age $(\mathrm{yr})$ & 67.0 & 70.0 & 0.530 \\
Gender $(\mathrm{M}: \mathrm{F})$ & $7: 3$ & $9: 1$ & 0.290 \\
Weight $(\mathrm{kg})$ & 75.3 & 84.9 & 0.067 \\
Height $(\mathrm{cm})$ & 171.6 & 177.0 & 0.170 \\
Degree of coronal deformity $\left({ }^{\circ}\right)$ & 7.2 & 6.0 & 0.500 \\
Preoperative range of flexion $\left({ }^{\circ}\right)$ & 107.1 & 111.0 & 0.430 \\
\hline
\end{tabular}

Table 2. Inpatient Outcomes for the Tourniquet and No Tourniquet Patient Groups

\begin{tabular}{lccc}
\hline \multicolumn{1}{c}{ Parameter } & Tourniquet & No tourniquet & p-value \\
\hline Total drainage post-operatively $(\mathrm{mL})$ & 457.1 & 436.2 & 0.670 \\
Transfusion requirements & 3 patients & 0 patients & 0.050 \\
Morphine equivalents using patient controlled analgesia $(\mathrm{mL})$ & 870 & 914 & 0.815 \\
Days to discharge & 7.3 & 5.3 & 0.230 \\
\hline
\end{tabular}




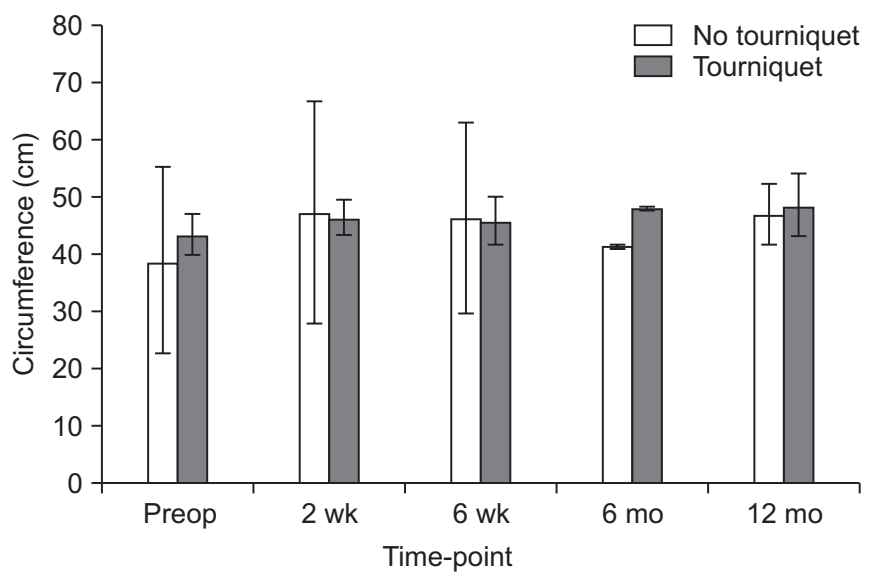

Fig. 3. Thigh circumference comparison between the tourniquet and no tourniquet groups. Preop: preoperative.

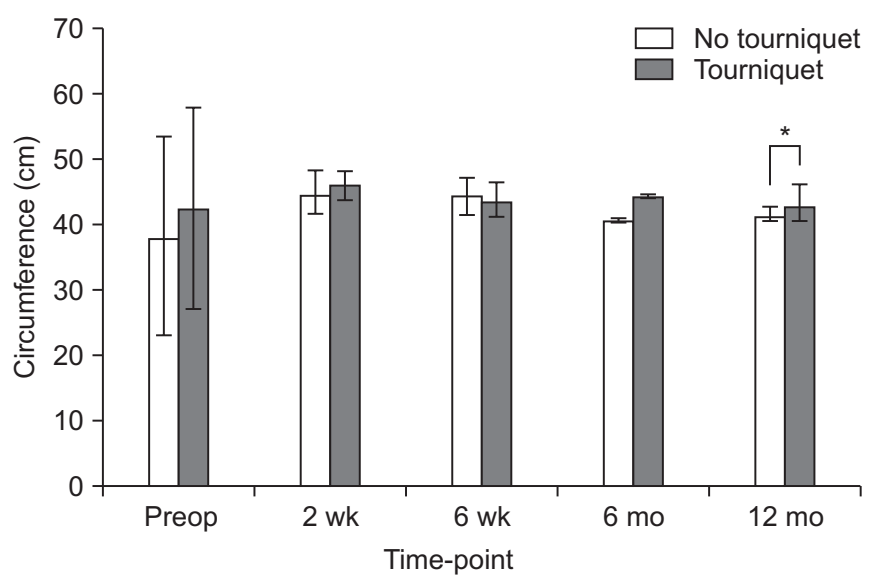

Fig. 4. Knee circumferences for the tourniquet and no tourniquet groups.

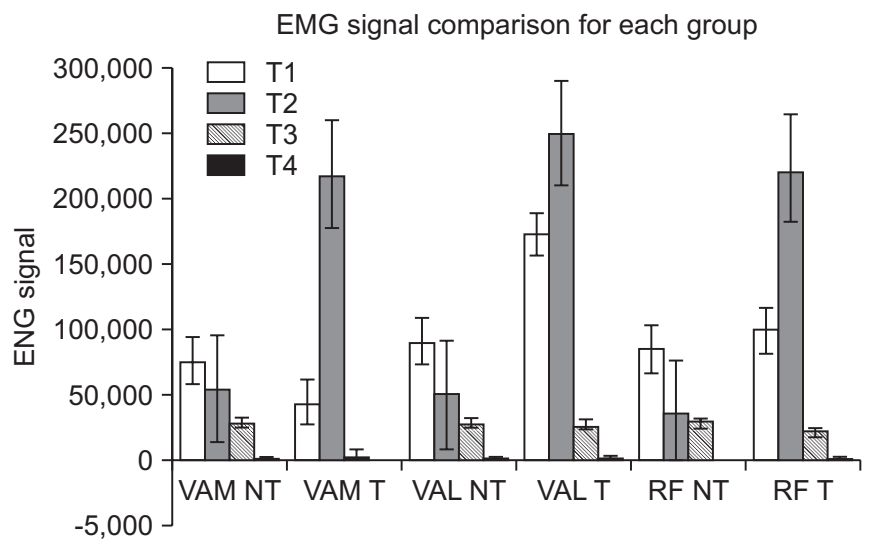

Fig. 5. Surface electromyography (EMG) in the tourniquet (T) and no tourniquet (NT) groups. T1 represents the pre-operative measurements, with T2, T3 and T4 measurements are at the 6 week, 6 month and 2 month follow-up periods. VAM, VAL and RF stand for vastus medialis, vastus lateralis and rectus femoris respectively. VAM: vastus medialis, VAL: vastus lateralis, RF: rectus femoris.

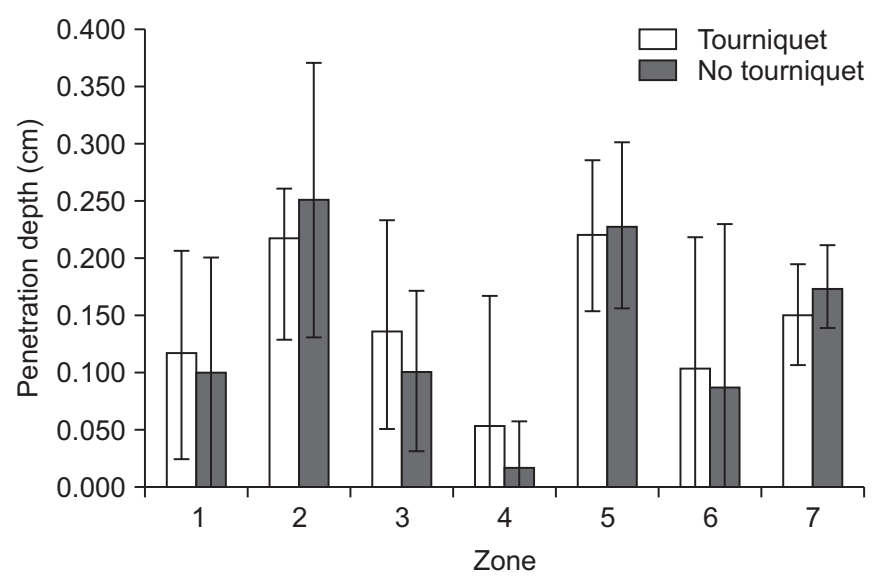

Fig. 6. Femoral cement penetration in Knee Society radiographic zone.

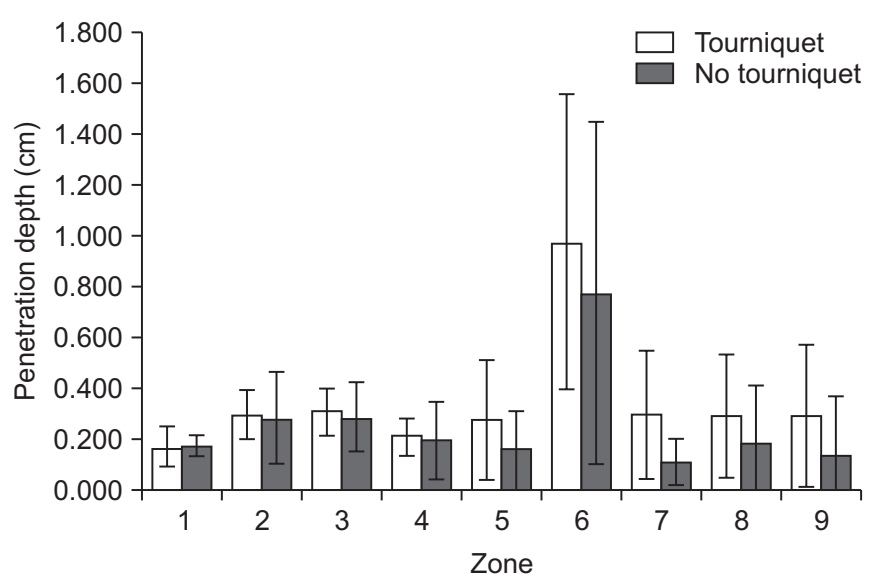

Fig. 7. Tibial cement penetration in Knee Society anteroposterior radiographic zones.

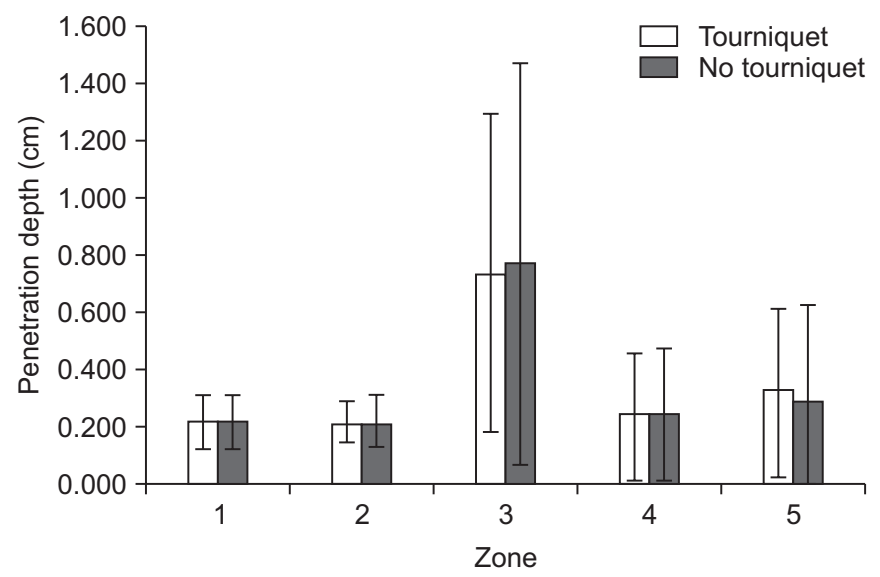

Fig. 8. Tibial cement penetration in Knee Society mediolateral radiographic zones. 
significance. Oxford knee scores and range of motion were not significantly different between the tourniquet and no tourniquet groups at all follow-up points up to 12 months. Thigh and knee circumference measurements are shown in Figs. 3 and 4, respectively.

The tourniquet group showed significantly less muscle activity as measured by surface EMG than the no tourniquet group, as shown in Fig. 5. However, from 6 months post-operatively, the energy output achieved was similar between the two groups. Quadriceps EMG function returned to equivalency with the non-operative leg and between the tourniquet and no tourniquet patients by 12 months post-operatively.

At twelve months post-operatively, digital radiographs showed no significant difference in cement mantle thickness or the presence of radiolucency at the bone-prosthetic interface between the tourniquet and no tourniquet groups. This held true for all zones of the femur and tibia as shown in Figs. 6-8.

\section{Discussion}

Previous studies specifically comparing tourniquet use with no tourniquet in TKA have reported mixed conclusions ${ }^{17,18)}$. AbdelSalam and Eyres ${ }^{12)}$ found less pain, earlier straight-leg raise and knee flexion, less wound infections and deep venous thromboses without a tourniquet. In contrast, Wakankar et al. ${ }^{19)}$ observed no real difference and concluded the use of a tourniquet was safe. Vandenbussche et al.9) found only very small benefits of not using a tourniquet in terms of pain and knee flexion in the early postoperative period. Ledin et al..$^{20)}$ found significantly less pain and 11 degrees more flexion at 2 years in the no tourniquet group. More recently Tai et al. ${ }^{21)}$ demonstrated the tourniquet was beneficial in reducing blood loss, postoperative inflammation and muscle damage as measured by C-reactive protein and creatine phosphokinase levels. No previous studies have assessed the effect of tourniquet use on the bone-cement interface or long-term outcome.

Our study showed the use of a tourniquet does have a detrimental effect on the quadriceps muscle and patient's recovery following TKA. Pain scores were higher in the tourniquet group and reached statistical significance for days 2 and 4 postoperatively. The tourniquet, therefore, appears to be a significant source of post-operative pain. We found no difference in postoperative blood drainage, Oxford knee score or range of motion. Three patients in the tourniquet group required a transfusion, compared to none in the no tourniquet group. We acknowledge many patient factors determine transfusion need but were unable to analyse for this. Whilst the tourniquet controls intra-operative blood loss, it does not stop post-operative blood loss or reduce overall blood loss.

We also demonstrated the use of a tourniquet results in significant reduction in quadriceps function. Using surface EMG measurements, the patients without a tourniquet had superior quadriceps energy output. The difference in quadriceps function took between 6 to 12 months to return to equivalent levels compared to the patients' contra-lateral control leg. Our initial hypothesis was the quadriceps muscle would be permanently damaged by tourniquet-induced ischemia, leading to long-term atrophy and weakness but this was not shown in our study. The quadriceps muscle appeared to recover and return to normal between 6 to 12 months post-operatively.

The only demonstrable detrimental effect of not using a tourniquet in our study was an increased operative time of 13 minutes. Not using a tourniquet does impede the bloodless surgical field and requires more meticulous haemostasis during exposure and soft tissue release. However, there were no complications directly related to not using the tourniquet in our study. In particular, none of our patients sustained inadvertent soft tissue injury or damage. Another postulated risk associated with not using a tourniquet is the bone bed may have blood, debris and fat on its surface, compromising cementation. A significant finding of our study was no difference between the tourniquet and no tourniquet groups with regard to cement interface quality or incidence of bone-cement radiolucency in any of the Knee Society score radiographic zones for the femur or tibia at 12 months. We specifically analysed the prosthesis cement bone interface using fluoroscopically guided radiographs and a blinded observer. We believe cement mantle quality is a function more of cementing technique rather than use of a tourniquet. Cleaning the bone surfaces using pulsatile lavage and pressurising the cement into the bone are more important determinants of cement interface quality. Not using a tourniquet does not appear to compromise the bone cement interface and therefore we believe should have no effect on long-term outcome or fixation.

A weakness of our study is the small number of patients. Power calculation with 10 patients in each group was $65 \%$, raising the risk of type II statistical errors. The lower power of the study, we believe, may be one of the reasons why the pain scores were not significantly different on all of the post-operative days and why hospital stay was not significantly shorter. Calculation prior to study commencement postulated 30 patients per group is required to give a power of $95 \%$. However, following study commencement, patient recruitment was difficult as potential pa- 
tients were informed they would not receive any form of regional or local anaesthesia. Many patients elected not to be involved in the study for fear of significant initial post-operative pain. We elected not to use any form of regional anaesthesia in our study that may confound pain scores or EMG results. Following a prolonged recruitment period, analysis of the available results already revealed differences in EMG readings and no difference in radiographic analysis and hence patient recruitment was ceased. Despite the low numbers, we still feel the findings of our study are relevant and valid. The strengths of our study include that it is a randomised controlled trial of a non-selected study population with few exclusion criteria. We used several highly objective outcome measures such as quadriceps EMG activity and radiographic cement interface quality.

Recently some surgeons advocate using lower tourniquet pressures to reduce the risk of post-operative pain and complications. Setting the tourniquet cuff pressure on the basis of systolic blood pressure plus a margin of $100 \mathrm{mmHg}$ has been reported to reduce cuff pressure and early post-operative pain ${ }^{22)}$. Olivecrona et $\mathrm{al}^{23)}$ in a randomised study demonstrated the limb-occlusionpressure method reduces the tourniquet cuff pressure used without compromising the quality of the bloodless field. No advantage was found with the lower cuff pressure with regards to postoperative pain, knee range of motion or complications. However, an important finding was patients with a cuff pressure less than $225 \mathrm{mmHg}$ had no post-operative infections and a lower rate of wound complications.

Another strategy is to reduce the tourniquet time by deflating the tourniquet prior to wound closure or to limit tourniquet use to specific steps of the procedure such as during cementation. The study by Tarwala et al. ${ }^{24)}$ compared tourniquet use for cementation only versus the entire operation. They found no important clinical differences between the two groups and their preference is to limit use of the tourniquet to the cementation portion of the procedure. We acknowledge the detrimental effect of the tourniquet is time-dependent ${ }^{25)}$ and our tourniquet inflation time was quite long at 83 minutes. Future research into optimal cuff pressure and tourniquet times will be necessary to determine the ideal patient individualised strategy for tourniquet use in TKA. One disadvantage common to all tourniquet strategies is in some patients the tourniquet may be difficult to effectively apply. The fit of the cuff to the limb, tightness of cuff application, and properties of the patient's soft tissues all affect how well the tourniquet will function. By not using a tourniquet altogether this variable is removed.

\section{Conclusions}

With the number available, our study suggests tourniquet use in TKA results in higher pain scores in the initial post-operative period and a reduction in quadriceps function for the first 6 months post-operatively. Use of a tourniquet had no effect on the prosthetic cement interface at 12 months post-operatively. We believe not using a tourniquet in TKA may be advantageous to the patient's recovery, without any obvious detrimental effect. Further studies are required to clarify the ideal strategy for tourniquet use in TKA.

\section{Conflict of Interest}

No potential conflict of interest relevant to this article was reported.

\section{Acknowledgments}

We would like to acknowledge and thank Smith and Nephew, Australia for their financial support in conducting the study.

\section{References}

1. McAllister CM, Stepanian JD. The impact of minimally invasive surgical techniques on early range of motion after primary total knee arthroplasty. J Arthroplasty. 2008;23:108.

2. Thienpont E. Faster recovery after minimally invasive surgery in total knee arthroplasty. Knee Surg Sports Traumatol Arthrosc. 2013;21:2412-7.

3. Kim YH, Kim JS, Kim DY. Clinical outcome and rate of complications after primary total knee replacement performed with quadriceps-sparing or standard arthrotomy. J Bone Joint Surg Br. 2007;89:467-70.

4. Huang HT, Su JY, Chang JK, Chen CH, Wang GJ. The early clinical outcome of minimally invasive quadriceps-sparing total knee arthroplasty: report of a 2-year follow-up. J Arthroplasty. 2007;22:1007-12.

5. Li B, Wen Y, Wu H, Qian Q, Lin X, Zhao H. The effect of tourniquet use on hidden blood loss in total knee arthroplasty. Int Orthop. 2009;33:1263-8.

6. Clarke MT, Longstaff L, Edwards D, Rushton N. Tourniquetinduced wound hypoxia after total knee replacement. J Bone Joint Surg Br. 2001;83:40-4.

7. Wakai A, Winter DC, Street JT, Redmond PH. Pneumatic 
tourniquets in extremity surgery. J Am Acad Orthop Surg. 2001;9:345-51.

8. Noordin S, McEwen JA, Kragh JF Jr, Eisen A, Masri BA. Surgical tourniquets in orthopaedics. J Bone Joint Surg Am. 2009;91:2958-67.

9. Vandenbussche E, Duranthon LD, Couturier M, Pidhorz L, Augereau B. The effect of tourniquet use in total knee arthroplasty. Int Orthop. 2002;26:306-9.

10. Estebe JP, Davies JM, Richebe P. The pneumatic tourniquet: mechanical, ischaemia-reperfusion and systemic effects. Eur J Anaesthesiol. 2011;28:404-11.

11. Husted H, Toftgaard Jensen T. Influence of the pneumatic tourniquet on patella tracking in total knee arthroplasty: a prospective randomized study in 100 patients. J Arthroplasty. 2005;20:694-7.

12. Abdel-Salam A, Eyres KS. Effects of tourniquet during total knee arthroplasty: a prospective randomised study. J Bone Joint Surg Br. 1995;77:250-3.

13. Shenton DW, Spitzer SA, Mulrennan BM. Tourniquet-induced rhabdomyolysis: a case report. J Bone Joint Surg Am. 1990;72:1405-6.

14. Palmer SH, Graham G. Tourniquet-induced rhabdomyolysis after total knee replacement. Ann R Coll Surg Engl. 1994;76: 416-7.

15. Appell HJ, Glöser S, Duarte JA, Zellner A, Soares JM. Skeletal muscle damage during tourniquet-induced ischaemia: the initial step towards atrophy after orthopaedic surgery? Eur J Appl Physiol Occup Physiol. 1993;67:342-7.

16. Saunders KC, Louis DL, Weingarden SI, Waylonis GW. Effect of tourniquet time on postoperative quadriceps function. Clin Orthop Relat Res. 1979;(143):194-9.

17. Tai TW, Lin CJ, Jou IM, Chang CW, Lai KA, Yang CY. Tour- niquet use in total knee arthroplasty: a meta-analysis. Knee Surg Sports Traumatol Arthrosc. 2011;19:1121-30.

18. Smith TO, Hing CB. Is a tourniquet beneficial in total knee replacement surgery? A meta-analysis and systematic review. Knee. 2010;17:141-7.

19. Wakankar HM, Nicholl JE, Koka R, D’Arcy JC. The tourniquet in total knee arthroplasty: a prospective, randomised study. J Bone Joint Surg Br. 1999;81:30-3.

20. Ledin H, Aspenberg P, Good L. Tourniquet use in total knee replacement does not improve fixation, but appears to reduce final range of motion. Acta Orthop. 2012;83:499-503.

21. Tai TW, Chang CW, Lai KA, Lin CJ, Yang CY. Effects of tourniquet use on blood loss and soft-tissue damage in total knee arthroplasty: a randomized controlled trial. J Bone Joint Surg Am. 2012;94:2209-15.

22. Worland RL, Arredondo J, Angles F, Lopez-Jimenez F, Jessup DE. Thigh pain following tourniquet application in simultaneous bilateral total knee replacement arthroplasty. J Arthroplasty. 1997;12:848-52.

23. Olivecrona C, Ponzer S, Hamberg P, Blomfeldt R. Lower tourniquet cuff pressure reduces postoperative wound complications after total knee arthroplasty: a randomized controlled study of 164 patients. J Bone Joint Surg Am. 2012;94: 2216-21.

24. Tarwala R, Dorr LD, Gilbert PK, Wan Z, Long WT. Tourniquet use during cementation only during total knee arthroplasty: a randomized trial. Clin Orthop Relat Res. 2014;472: 169-74.

25. Butt U, Ahmad R, Aspros D, Bannister GC. Factors affecting wound ooze in total knee replacement. Ann R Coll Surg Engl. 2011;93:54-6. 